\title{
Optimizing the computational simulations of air- flammable gas explosions using HPC and Ansys software
}

\author{
Laurenţiu Munteanu*, Marius Cornel Şuvar, and Ligia Ioana Tuhuţ \\ National Institute for Research and Development in Mine Safety and Protection to Explosion \\ INSEMEX Petrosani, 32-34 G-ral Vasile Milea Street, Petroşani 332047, Romania
}

\begin{abstract}
The purpose of this scientific work is to improve computer simulations of flammable air-gas explosions with HPC systems.

Computational Fluid Dynamics (CFD) is increasingly used for obtaining variable values of fluid flow areas, respectively for the manner in which fluids react with limited surfaces.

For a separate analysis of liquids and gases is used CFD, and for more realistic results is used the multi-phase method performed by ANSYS Fluent, which improves the calculation scalability and power.

For increasing the processing speed for complex analyses, with multiple geometries and fine meshes, ANSYS provides the user HPC (High Performance Computing) tools applicable for structural, thermal, electromagnetic, fluid dynamics and explicit dynamics solvers. HPC configuration is characterized by a good scalability, having the capacity for future extension of cores or processors.

For decreasing the computational simulation time for explosions, the proposed solution consists in running complex simulations on the servers of a HPC cluster. In this way is provided the possibility for a parallel or distributed running on one or several calculation systems. Using HPC along ANSYS applications may be activated by GPU acceleration, while other applications are limited to processing using CPUs. INSEMEX develops technical investigations of explosions and fires occurred in the industrial or civilian field, in compliance with Government Decision $1461 / 2006$, based on the verification of scenarios using virtual computational simulations.
\end{abstract}

\section{Introduction}

Explosions in air-methane mixture are specific to mining industry and are the most common. These incidents lead to significant economic losses and a large number of victims. [1]

Physical phenomena associated with explosions, such as releases of gas and liquid, heat and mass transfer, body movement, chemical reactions, fluid-structure interactions as well as acoustic waves can be simulated by computer modelling.

\footnotetext{
${ }^{*}$ Corresponding author: laurentiu.munteanu@insemex.ro
} 
Evolution of computing technique and emergence of new generation computers, has led to development of methods for studying the combustion mechanism and explosion of gas mixtures, allowing them to be studied in depth through computer modelling and simulation.

Since the 1970s a new branch of fluid mechanics, CFD, has been developed, which uses algorithms, numerical methods and calculations to model and solve problems in which fluid flows occur. [1]

To solve complex linear and nonlinear, static and dynamic analysis problems, the ANSYS application suite can be used with HPC tools. [5]

\section{Materials and methods}

\subsection{Notions related to explosive flammable air-gas mixture}

Within industrial activities carried out in explosive and/or toxic atmospheres in the underground/surface, because of specific risks, accidents can occur generating negative effects in terms of human and material losses, affecting civil society and the environment. [2]

The danger of explosion is associated with flammable materials and substances, extracted, processed, stored or used by industrial or domestic installations and equipment. Some of these materials and substances may be subjected to combustion processes in the air. These processes are often accompanied by the release of considerable amounts of heat and may be associated with an accumulation of pressure and air pollution. [1]

Knowledge of explosion limits is of particular importance in mining, chemical and petrochemical industries, for safe operation of installations.

If in the atmosphere, methane has a concentration lower than the lower ignition limit of $5.0 \%$, an explosion cannot occur even if there is a source of ignition. [7]

Temperature, pressure and oxygen concentration in the air also influence flammability limits.

The greatest danger regarding methane, an explosive gas, is its property to form explosive mixtures with air, following the reaction: [3]

$$
\mathrm{CH}_{4}+\left(2 \mathrm{O}_{2}+8 \mathrm{~N}_{2}\right)=\mathrm{CO}_{2}+2 \mathrm{H}_{2} \mathrm{O}+8 \mathrm{~N}_{2}
$$

resulting a volume of methane that burns completely and consumes all the oxygen in ten volumes of air.

Methods used to control the concentration of a potentially explosive gas or vapour include the use of purifying gas, of non-reactive gas, such as nitrogen or argon, to dilute the explosive gas before coming into contact with air. [3]

Table 1. Mixing limits for flammable gas combustion (in\% volume) and ignition temperatures [3]

\begin{tabular}{|l|c|c|c|c|c|c|c|}
\hline \multicolumn{2}{|c|}{} & \multicolumn{2}{c|}{$\begin{array}{c}\text { Limit in atmospheric } \\
\text { air }\end{array}$} & \multicolumn{2}{c|}{ Limit in oxygen } & $\begin{array}{c}\text { In } \\
\text { atmospheric } \\
\text { air }\end{array}$ & $\begin{array}{c}\text { In } \\
\text { pure } \\
\text { oxygen }\end{array}$ \\
\hline \multicolumn{1}{|c|}{ Name } & Symbol & Inferior & Superior & Inferior & Superior & {$\left[{ }^{\circ} \mathbf{C}\right]$} & {$\left[{ }^{\circ} \mathbf{C}\right]$} \\
\hline Hydrogen & $\mathrm{H}_{2}$ & 4,0 & 74,2 & 4,0 & 94,0 & 570 & 560 \\
\hline Carbon oxide & $\mathrm{CO}$ & 12,5 & 74,0 & 15,5 & 94,0 & 610 & 590 \\
\hline Methane & $\mathrm{CH}_{4}$ & 5,1 & 15,0 & 5,0 & 65,0 & 650 & 535 \\
\hline Acetylene & $\mathrm{C}_{2} \mathrm{H}_{2}$ & 2,3 & 81,0 & 2,8 & 93,0 & 335 & 295 \\
\hline Ethylene & $\mathrm{C}_{2} \mathrm{H}_{4}$ & 3,0 & 33,5 & 3,0 & 80,0 & 540 & 485 \\
\hline Ethane & $\mathrm{C}_{2} \mathrm{H}_{6}$ & 3,0 & 14,0 & 3,9 & 50,5 & 520 & - \\
\hline Propane & $\mathrm{C}_{3} \mathrm{H}_{8}$ & 2,1 & 9,3 & 2,2 & 45,0 & 480 & 470 \\
\hline Butane & $\mathrm{C}_{4} \mathrm{H}_{10}$ & 1,7 & 8,4 & 1,8 & 40,0 & 460 & 280 \\
\hline
\end{tabular}




\subsection{Computerized fluid dynamics (CFD). ANSYS Fluent}

For a better understanding of phenomena analysed and to facilitate interpretation of physical experiments, numerical analysis is used. With the use of CFD from ANSYS suite, phenomena or processes can be numerically simulated, in order to establish indices or characteristics that are very difficult to be drawn by physical experiments. [1]

Nowadays CFD analysis techniques play an increasingly important role in activities such as: research, design, production, construction. [1]

In the ANSYS Multiphysics package, numerical modelling of fluid dynamics can be achieved through specialized applications such as ANSYS CFX, ANSYS ICEM CFD, FLUENT, POLYFLOW. With ANSYS CFX it is possible to analyse the fluid flow in the context of design-manufacturing cycle and, as well, to study the manner in which rotary machine's performances can be improved. [9] ANSYS ICEM CFD is used for conceptual and preliminary aerodynamic design. FLUENT is a flexible CFD package, used for simulations of any complexity that offers a complete range of physical models that can be used for a wide range of applications, from various industries. POLYFLOW is an advanced tool that includes a very wide range of thick-elastic fluid models, being used especially in case of analyses performed in the field of polymer processing and in many applications in the field of processing of materials. [1]

ANSYS CFX and FLUENT applications are used for the computer simulation of flammable air-gas explosions. [1]

With the use of ANSYS Fluent application, different methods of virtual modelling of explosions in confined spaces can be analysed, models that allow definition of translation / rotation movements of digitization networks. A digitization network as it is shown in figure 1 is divided into control volumes called cells. Each cell is defined by a set of nodes, a cell centre and the faces that limit the cell. [1]

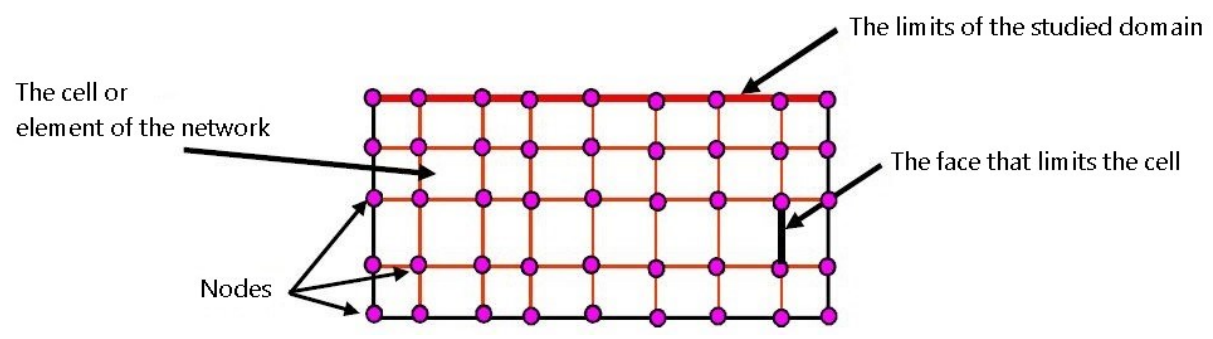

Fig. 1. Components of a digitization network

Uploading a digitization model in the FLUENT program has the effect of defining the geometric entities according to the application code, resulting in the boundaries and surfaces of the studied domain. [1]

The ANSYS Fluent application offers a wide range of functions and macros to facilitate the programming of user-defined functions (UDFs) required in the use of CFD objects, as defined in the program. [1]

The user can benefit from programming languages, for defining the desired functions such as C, FORTRAN, SCHEME, CFX Command Language (for post-processing), and by using the own command lines, text commands and system commands can be performed. [1]

Within the project accomplished by INCD INSEMEX in 2014, named "PN 07-45-02-46 - Optimization of computer simulation for explosions of air-methane mixtures through ANSYS CFX and FLUENT applications" 24 computer simulations of explosion of a stoichiometric air-methane mixture in a confined space were performed.

Performing tests on different methods of dynamic digitization networks, in which the geometric model is represented by a rectangular tube, according figure 2 and figure 3 sealed 
at both ends, the following result was obtained: the explosion produced at one end of the enclosed space, generates an internal pressure acting on the tube's walls with a force directly proportional to their surface. [1]

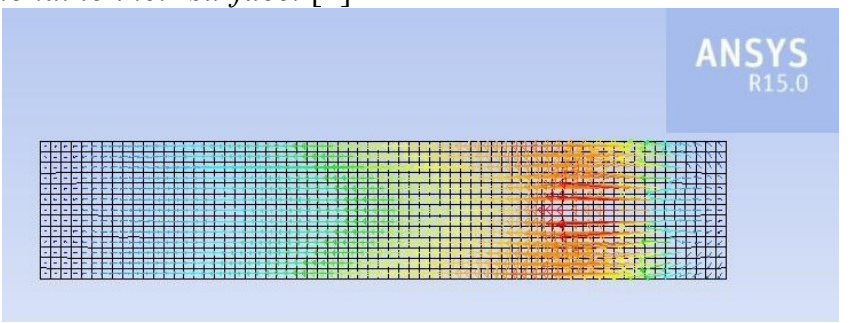

Fig. 2. Simulation of the explosion in the tube, without deforming the walls

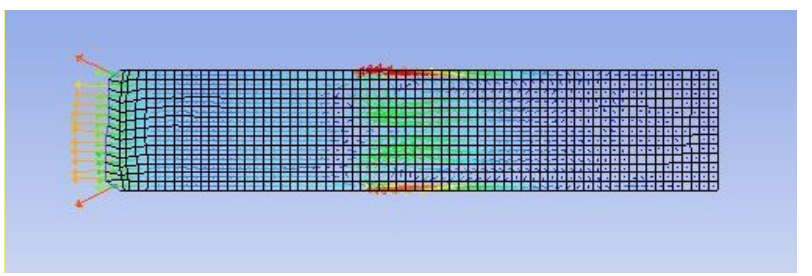

Fig. 3. The moment tube's walls breakage

Following the breakage of the wall and release of gas into the outer atmosphere, there's a sharp decrease in the pressure on the border surface, according figure 2 and figure 3 a pulsating phenomenon of gas inlet and outlet occurring in the tube, pressure gradients taking decreasing values. [1]

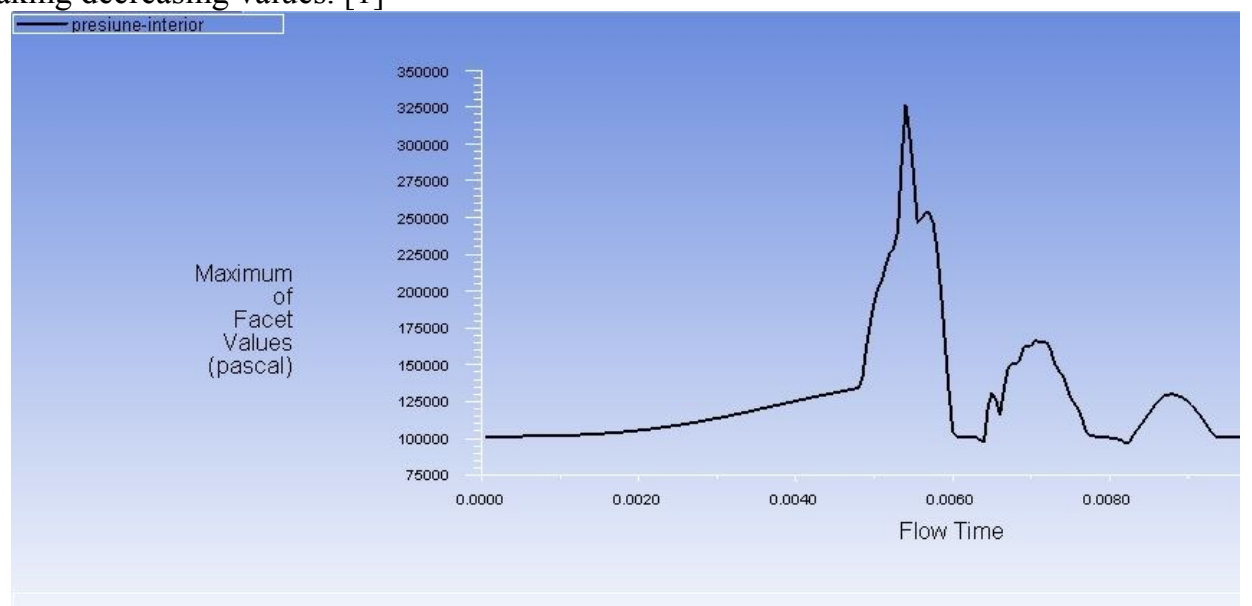

Fig. 4. Evolution of the internal pressure during its equalization with the external pressure

Thus, the model created for simulating gas explosions in confined spaces, based on the use of user-defined functions (UDF), accurately describes the evolution of pressure, velocity, etc. gradients during the event. [1]

The model can be used both in the industrial environment, for simulation of explosion scenarios in containers, pipes, production halls, as well as for studying the behaviour of structural elements of residential buildings, storage spaces in gas explosions. [1] 


\subsection{The importance and the weaknesses of the system HPC in explosion simulation}

The European Union calls high performance computing a strategic resource, for the future of Europe, essential for development, innovation and the labour market. [8]

The use of parallel and distributed numerical calculation methods, using computing resources of the existing HPC system, has led to a significant reduction of time for settling all case studies, which is a special advantage in the case of technical expertise of explosion events, like those developed within INCD INSEMEX. Computer simulations of these events are very useful in analysing the scenarios and hypotheses elaborated, contributing to consolidation of conclusions regarding the causes of event's occurrence and its mechanism. [2]

By using HPC tools, it is possible to extend the integrated research platform based on new IT technologies and to increase the processing capacity needed to solve complex research topics.

Also, we emphasize other benefits:

- HPC can be used in many areas, such as mechanical engineering (design and production), electronics design, natural sciences (including forecasts and weather maps or natural resource management), medicine, finance and investment (e.g. risk analysis), cyber security and defence, as well as in everything related to scientific research. [6]

- Mathematical approach to the phenomenology of fast combustion in the investigation of events generated by explosions, resulting in material damage and loss of human life, respectively in the evaluation of the explosion risk in case of work / technological processes carried out in potentially explosive atmospheres. [4]

- Identification of explosion mechanisms for events in domestic / industrial environments and subsequent elaboration of measures to prevent similar dangerous situations for domestic/industrial gas supply applications and installations. [1]

- Cost reduction for physical experiments. [1]

The weaknesses of implementing an HPC cluster are:

- significant increase in processing speed requires the purchase of more hardware devices, respectively HPC nodes with specialized software;

- as the number of nodes increases, the total price of the HPC cluster increases and implicitly the electricity consumption;

- there are needed more ventilations for cooling equipment and the result is increasing the noise level;

- racks should be placed in special rooms with rigorous environmental control.

\subsection{The presentation of technical expertise}

Technical expertise represents the basis for researches carried out for development of new concepts, methods, processes and technologies in order to know, evaluate, prevent and reduce risk factors.

INCD INSEMEX Petrosani carries out activities regarding the development of judicial and extrajudicial technical expertise of events produced by explosions generated by explosive mixtures of air-flammable substances (flammable vapours, mists, gases) or explosive substances with intrinsic properties (explosives or pyrotechnic mixtures).

The ANSYS software package is used in all technical expertise of explosion events, accurately establishing the facts or circumstances of the case.

One of the important events in which the computer simulation was carried out, following the explosion of a natural gas transport pipeline, is the one produced between the natural gas-air mixture and the short circuit between the cables of the road electric network. 
The image from the place can be seen in fig. 5 and highlighted by: sectioning of a metallic pipe located at the base of a deep crater of $3 \mathrm{~m}$, burning of the neighboring vegetation on a distance of $70 \mathrm{~m}$, damage of the nearby buildings, destruction by incineration of vehicles and electrical installations.

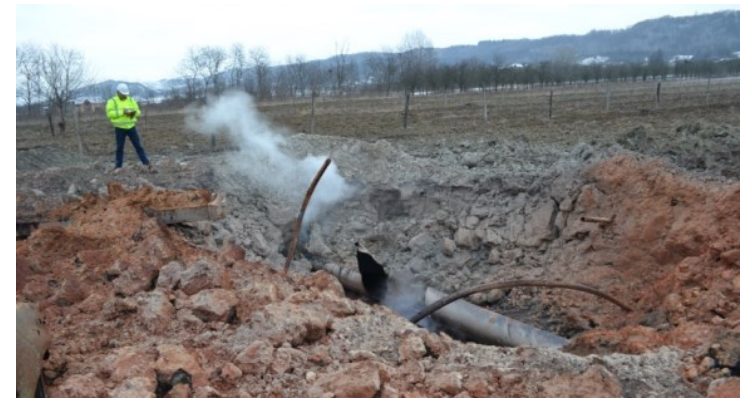

Fig. 5. The crater formed after the explosion with the unevenly sectioned pipe

By computer simulation the pressure and external corrosion of the pipe were established and outlines of the flame wave, according figure 6 , respectively the pressure wave, could be visualized through colour contours, providing important information on the explosion's mechanism.

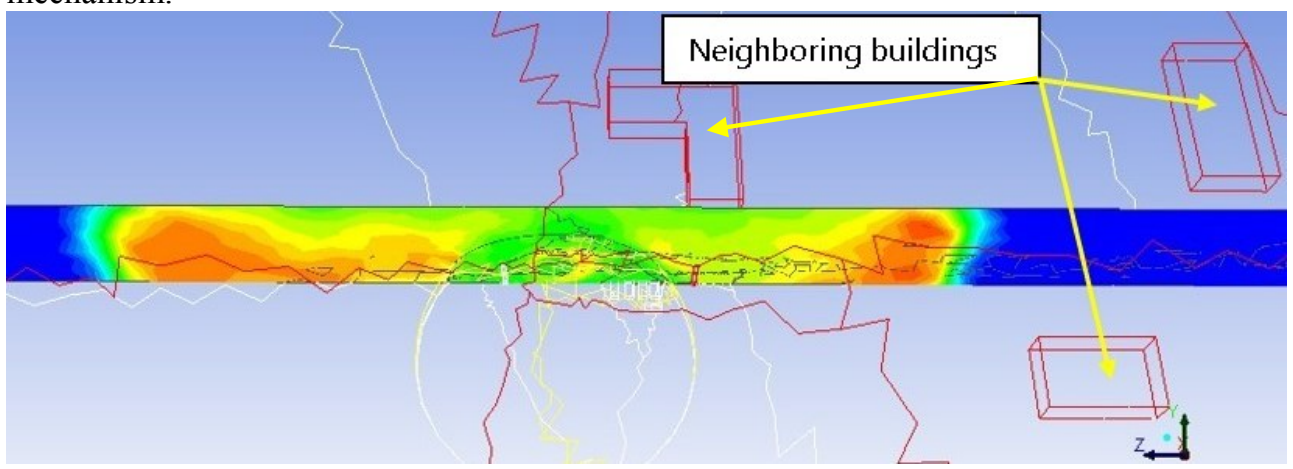

Fig. 6. The flame front spreads to nearby buildings

If new relevant elements and explicit evidence come up, computer simulations can easily be re-evaluated, for analysis and hypotheses for mulated for pre-processing and postprocessing of their results.

INCD INSEMEX has found that the most common cause of explosion incidents is the occurrence of cracks in the underground gas pipelines, which generates gas migration towards properties of assessed buildings.

\section{Results and conclusions}

In many disaster scenarios, destruction is so severe that rebuilding the event is difficult. ANSYS software allows virtual modelling in different structures and tests the impact of environmental and destructive forces, whenever necessary to fully understand effects of the disaster.

Modelling combustion processes by using CFD, for complex, real scenarios, generates very long performance times, of days or even weeks. In developing design studies or postevent technical expertise, time is a very valuable parameter, engineers must provide maximum accuracy results in the shortest possible time. In this situation, the use of HPC systems is the best solution from a technical point of view, although it is not always 
accessible, because of high costs. By using the UDF functions, along with methods of dynamic digitization networks, the diversity of scenarios that can be simulated in both industrial and residential environment increases.

The hardware platform used by INCD INSEMEX Petrosani as it is shown in figure 7 is of HPC cluster type, consisting of 1 Master node server and 10 Compute Node servers.
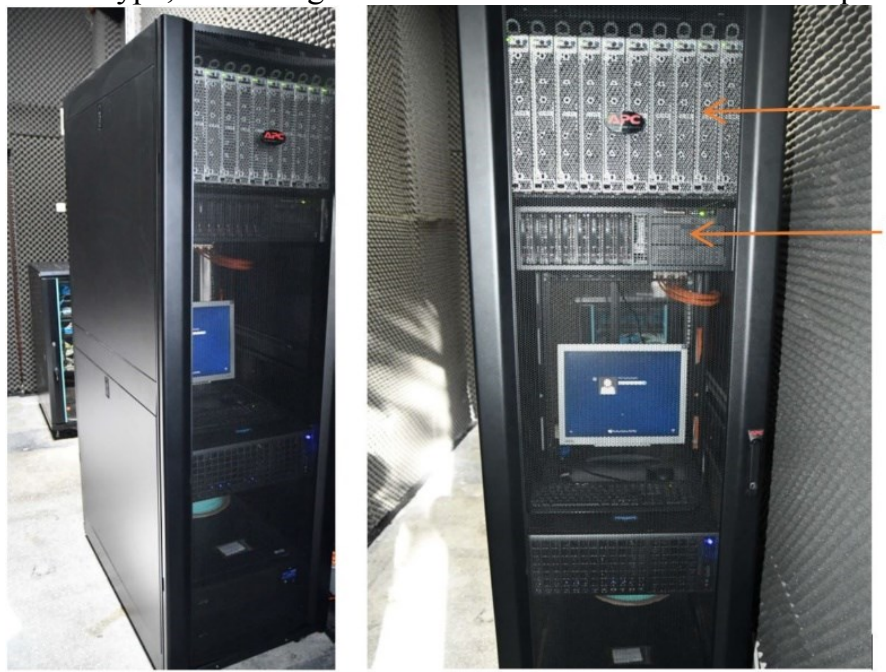

Compute

nodes

Master node

Fig. 7. The HPC Cluster from INCD Insemex Petrosani

INCD Insemex has an optimized HPC configuration that contains two types of nodes: a management node (also known as the admin node or head node), which is the central administration node of the cluster through which the other nodes are installed, managed and monitored and computing nodes, which perform the tasks and calculations required by the admin node.

Before using computer simulation for technical explosions' expertise of flammable airgas mixtures, INCD Petrosani used empirical methods, scenarios' simulations by making models-layouts in laboratories and it prepared around 4 technical expertise per year. Using the field of numerical modeling and computer simulations, INCD Petrosani prepares around 12 of such technical expertise per year. The computerized simulation made it possible to increase the accuracy of the results and to approximate some explosion parameters (temperature, pressure, fuel gas concentrations) which cannot be determined during the field investigations.

\section{References}

1. PN 07-45-02-46, Nucleu Program INCD INSEMEX, Optimization of computer simulation for explosions of air-methane mixtures through ANSYS CFX and FLUENT applications (2014)

2. PN 16-43-03-03, Nucleu Program INCD INSEMEX Research on fire computerized modelling using HPC systems (2016-2017)

3. D. Cioclea, Sectorial Project INCD INSEMEX Reducing the risk of explosion at the underground coal mines in Jiu Valley, through computerized management of the ventilation networks (2011)

4. PN 07-45-03-25, Nucleu Program INCD INSEMEX, Programming and using subroutines for moving digitization networks to solve computer fluid mechanics problems (2015)

5. D. G. Ionescu, introduction to fluid mechanics, Technical Publishing House, Bucharest (2005)

6. HPC computing. Available on https://ittrends.ro/2011/07/hpc-computing-la-un-alt-nivel/

7. Flammability limit. Available on https://en.wikipedia.org/wiki/Flammability_limit

8. HPC. Available on https://ec.europa.eu/digital-single-market/en/high-performance-computing

9. ANSYS FLUENT manual, made available by INAS S.A. 